\title{
Multilinguales
}

Multilinguales

12 | 2020

Varia 2019

\section{Vers une analyse logico-linguistique des structures de comparaison}

Towards a logic-linguistic analysis of comparison structures

\section{Amor tahar}

\section{(2) OpenEdition}

\section{Journals}

\section{Édition électronique}

URL : https://journals.openedition.org/multilinguales/4616

DOI : $10.4000 /$ multilinguales.4616

ISSN : 2335-1853

\section{Éditeur}

Université Abderrahmane Mira - Bejaia

\section{Référence électronique}

Amor tahar, « Vers une analyse logico-linguistique des structures de comparaison », Multilinguales [En ligne], 12 | 2020, mis en ligne le 14 février 2020, consulté le 30 juin 2021. URL : http:// journals.openedition.org/multilinguales/4616; DOI : https://doi.org/10.4000/multilinguales.4616

Ce document a été généré automatiquement le 30 juin 2021.

\section{(c) (i) (8)}

Multilinguales est mise à disposition selon les termes de la Licence Creative Commons Attribution Pas d'Utilisation Commerciale - Pas de Modification 4.0 International 


\title{
Vers une analyse logico-linguistique des structures de comparaison
}

Towards a logic-linguistic analysis of comparison structures

\author{
Amor tahar
}

1 Une tâche cognitive de base et un type très particulier des relations sémantiques, telle est la comparaison. Premièrement, la comparaison est une catégorie profondément syntaxique. Il n'y a pas de comparaison en dehors d'une référence, plus ou moins explicite à une échelle de valeurs. Les comparaisons morphologiques elles-mêmes n'expriment aucune comparaison, du moins jusqu'à ce qu'elles soient incluses dans des phrases. Et, deuxièmement, la proposition est formée par l'interaction de deux plans, l'un reflétant le lien de la proposition avec le monde objectif, l'autre, son lien avec le processus de réflexion : si la comparaison est une catégorie profondément syntaxique, elle n'est pas une catégorie linguistique proprement dite puisqu'elle est, avant tout, un processus logique permettant à un sujet pensant d'apprendre sur le monde. L'approche logico-linguistique permet donc d'étudier plus adéquatement les mécanismes de formation de structures syntaxiques avec la sémantique de la comparaison. J'essaie donc de présenter l'ontologie d'un cadre sémantique qui cherche à représenter la signification des structures comparatives en langage naturel, et qui modélise les comparaisons sous forme des paires prédicat-argument interconnectées avec des rôles sémantiques. Cette approche d'origine anglo-saxonne prend en charge non seulement les adjectifs, mais également les comparatifs adverbiaux, nominaux et verbaux.

\section{Comparaison : représentation logique de la signification}

2 Représenter le sens d'un texte est depuis longtemps un des champs d'investigation les plus propices pour la linguistique contemporaine, et diverses recherches récentes portant sur la compréhension du sens sémantiques telles que l'analyse sémantique automatique ont permis de dériver des modèles informatiques de signification. 
L'analyse sémantique profonde (par opposition à l'analyse sémantique superficielle, telle que l'étiquetage des rôles sémantiques) vise à associer une phrase en langage naturel à sa représentation formelle correspondante (Zelle et Mooney, 1996 ; Berant et Liang, 2014).

La comparaison est un moyen courant d'exprimer les différences de perceptions et d'autres propriétés vis-à-vis d'une entité. La comparaison peut se produire dans des structures très simples telles que "Paul est plus grand que Pierre " ou dans des constructions plus complexes telles que «La table est plus longue que le canapé est large ». Jusqu'à présent, la sémantique computationnelle des comparatifs et leur incidence sur la signification du texte n'ont pas été pleinement étudiées. C'est-à-dire que la différence existant entre la représentation sémantique et syntaxique des comparatifs n'est pas assez distinctive pour permettre une compréhension plus profonde d'une phrase. Par exemple, la représentation de signification sous forme logique générale de la phrase "Paul est plus grand que Sophie» ne rend pas complètement compte de la sémantique sous-jacente de l'adjectif " grand » et de ce que signifie être "grand». Un lecteur humain peut facilement en déduire que la taille de Paul est supérieure à celle de Sophie. Un autre exemple à prendre en considération est la phrase «Paul est grand », qui a fondamentalement la forme logique typique "grand (Paul) » comme une représentation très superficielle de la signification du prédicat « grand».

De même, un lecteur humain peut déduire que définir quelqu'un de "grand » dans un domaine de discours, et cela implique que cette personne est en quelque sorte "plus grande » que toutes autres personnes (en visant leur taille moyenne), cependant, la représentation sous forme logique typique plus tôt ne permet pas de telles inférences.

Dans cette communication, nous essayons de montrer un nouveau cadre pour la représentation sémantique et l'analyse computationnelle de la structure de comparaison en langage naturel. Ce cadre permet une représentation plus profonde de la sémantique des comparatifs, incluant tous les différents types de comparaisons entre comparatifs, superlatifs, équivalents, excessifs et assertifs, et la manière dont ils sont liés aux rôles sémantiques correspondants. En fait, il s'agit d'un jeu de données de structures comparatives annotées à l'aide d'une représentation automatique (automatisée) de la signification, ce qui permet des modèles d'apprentissage sur des constructions de comparaison. Nous proposons d'exposer cette nouvelle approche pour l'extraction automatique des structures de comparaison à partir d'un corpus de phrases ou d'un texte donné.

Une représentation sémantique de la comparaison exprimée par la phrase «L'équipement est trop vieux pour nous être d'une grande utilité » serait la suivante :

Figure 1 : Représentation sémantique de la comparaison

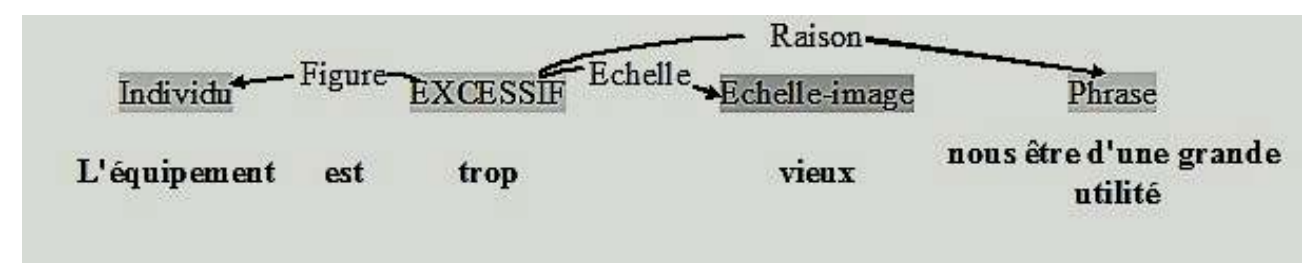

Puisqu'il n'est pas possible de dénombrer dans notre corpus tous les cas de figure, nous prenons particulièrement le cas de comparaison comme toute affirmation comparant 
deux entités ou plus, exprimant une sorte de mesure sur une échelle ou indiquant un certain degré de propriété mesurable. Les détails de ces variations seront discutés à la section 3.

\section{Positionnement théorique}

Dans cette section, il est nécessaire, avant toute chose de rendre compte du fondement théorique linguistique des structures comparatives en langage :

\subsection{Structures comparatives en langage}

7 La mesure en langage naturel s'exprime principalement dans des phrases ayant des morphèmes comparatifs tels que plus, moins, plus, aussi, assez, moins, etc. Les comparatifs peuvent être soit adjectivaux, adverbiaux, nominaux ou verbaux, c'est-àdire que le composant principal de la phrase effectuant la mesure peut avoir l'une ou l'autre de ces parties du discours.

Comparatifs adjectivaux : les exemples canoniques des phrases comparatives contiennent des adjectifs, par exemple "grand » ou « joli ». Même dans les comparatifs adjectivaux, il existe une grande variété de structures. Prenons les exemples suivants :

[1a] Marie est plus grande que Sophie.

[1b] Marie est trois pouces plus grande que Sophie.

[1b] Marie Marie est plus grande de cinq pieds.

8 La forme comparative de l'adjectif «grand» dans la phrase 1.a est considérée comme une expression désignant une relation plus que ( $>$ ) entre deux individus, «Marie » et «Sophie», sur l'échelle de» taille». L'analyse de degré théorique de tels adjectifs fait apparaître la notion d'adjectifs gradables (de nombreux facteurs décrivent des qualités qui peuvent être mesurées selon des degrés sur des échelles, telles que l'échelle de "taille", "beauté", "âge", etc.). Ces adjectifs peuvent être utilisés avec des morphèmes comparatifs, indiquant plus ou moins d'une qualité particulière sur une échelle. Les adjectifs gradables peuvent exprimer des relations spécifiques entre des individus sur une échelle, par exemple, dans la phrase $1 b$, Marie est plus grande que Sophie d'une mesure de trois pouces.

La comparaison à l'échelle ne concerne pas toujours deux individus. Par exemple, considérons la phrase $1 c$ qui indique qu'une comparaison est faite entre un individu et un point spécifique sur l'échelle de la « taille».

Tous les exemples précédents sont parmi les types les plus simples de structures comparatives utilisant des adjectifs. Prenons l'exemple suivant :

[2] Marie est plus grande que le lit est long.

Dans la phrase 2, nous avons un cas de sous-composition, dans lequel nous comparons "Marie» et "lit» selon deux dimensions différentes: la taille et la longueur. Chaque dimension fournit un degré, et les degrés sont finalement liés par la relation supérieure à ( \). L'extensibilité est connue pour être universelle dans la langue et une grande variété de phénomènes linguistiques peuvent être expliqués en termes de degrés et d'échelles (Solt, 2015).

La sémantique des échelles : une vue assez commune (Kennedy, 2007) est qu'une échelle S est un triple de la forme suivante : 
$\mathrm{S}=\{\mathrm{D},>, \mathrm{DIM}\}$

11 où $\mathrm{D}$ est un ensemble de degrés, > est une relation d'ordre sur D et DIM est la dimension de la mesure.

12 Les individus sont liés aux fonctions degré par mesure. Une fonction de mesure $\mu \mathrm{S}$ est la fonction qui mappe un individu $\mathrm{x}$ au degré sur l'échelle $\mathrm{S}$ qui représente la mesure de $\mathrm{x}$ par rapport à la dimension DIM. Par exemple, la fonction de mesure $\mu$ TAILLE est une fonction qui mappe les individus à leurs tailles respectives. Sous ce modèle, nous représentons la structure comparative des phrases 1a-1c comme suit :

[3a] $\mu$ TAILLE (Marie) > $\mu$ TAILLE (Sophie)

$[3 b] \mu$ TAILLE (Marie) $\geq \mu$ TAILLE (Sophie) +3 pouces

[3c] $\mu$ TAILLE (Marie) > 5 pieds

13 Dans ce modèle, nous pouvons également représenter la structure comparative de la phrase "Marie est grande", où il n'existe pas d'argument de degré explicite. Une hypothèse courante est que le rôle de degré est joué par un morphème de degré phonologiquement nul, qui désigne un seuil ou une norme de comparaison dépendant du contexte (Kennedy, 2007 ; Heim, 2007). Par exemple, dans un contexte spécifique où les hommes adultes en Amérique du Nord sont "grands", pourraient être interprétés comme ayant plus de 6 pieds.

Comparatifs non canoniques: Les structures comparatives peuvent également être verbales, nominales et adverbiales. Prenons les comparatifs verbaux suivants :

[4a] Les femmes mangeaient plus que les hommes.

[4b] Le lac s'est refroidi à plus de quatre degrés.

14 Il a été proposé (Wellwood et al., 2012) que les fonctions de mesure $(\mu)$ puissent être appliquées à la fois aux individus et aux événements, dans ce dernier cas, soit l'événement ou une entité en relation avec l'événement. L'interprétation comparative des deux phrases $4 a$ et $4 b$ est la suivante :

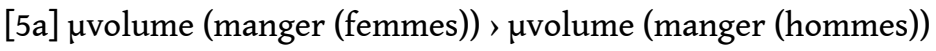

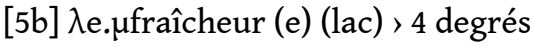

où s'est refroidi est interprété ici comme une fonction qui prend un événement e et un objet $\mathrm{x}$ (ici, « lac ») et renvoie un degré représentant le degré de modification de la fraîcheur de $\mathrm{x}$ du fait de sa participation à e. L'échelle sous-jacente des comparatifs verbaux est parfois ambiguë. Par exemple, dans la phrase 5a, il n'est pas clair si les femmes mangent plus en volume ou en quantité.

Les structures comparatives peuvent également être nominales. Prenons les phrases suivantes :

[6a] Plus de juniors que d'aînés sont venus à la cérémonie.

[6b] Nous avons acheté plus de lait que de vin.

16 La signification des phrases présentées ci-dessus doit également être mentionnée en degrés (Solt, 2015). Par conséquent, l'échelle pour la phrase comparative $6 a$ est le comptage numérique par des entiers et l'échelle pour la phrase $6 b$ correspond à une dimension de masse, ici peut-être un volume liquide. Les comparatifs adverbiaux partagent beaucoup de leurs caractéristiques avec les classes adjectivale et verbale, ce que nous ne développons pas plus par souci de brièveté. Par exemple, la phrase « Marie a couru plus vite que Pierre " est un exemple de comparaison adverbiale, dans laquelle l'attribut «vitesse » implicite de l'événement "course», associé à Marie et Pierre, est comparé. 


\subsection{Catégories de comparaison}

\section{de différence ou de similitude. Vous trouverez ci-après les principales catégories de} degrés de comparaison, ainsi que des exemples de phrases :

[7] comparatif

Marie est plus grande que Sophie.

Les chiens sont plus intelligents que les lapins.

[8] superlatif

Marie est la fille la plus grande de sa classe.

Les chiens sont les plus intelligents parmi les animaux de compagnie.

[9] équatif

Marie est aussi grande que Bill.

Les chiens sont aussi intelligents que les chats.

[10] excessif

Marie est trop courte pour le basketball.

Les chiens sont trop intelligents pour être dupes.

[11] actif

Marie est assez grande pour atteindre l'étagère.

Les chiens sont suffisamment intelligents pour retrouver le chemin de la maison.

\section{Approche automatique logico- sémantique de la comparaison}

18 Comme indiqué précédemment, le fait d'avoir une représentation profonde des structures de comparaison peut nous aider à construire des modèles informatiques de comparaison dans le langage naturel et à effectuer des tâches inférentielles dans différents domaines. Nous introduisons ici un nouveau cadre sémantique de comparaison. Ce cadre est basé sur les interprétations linguistiques présentées dans la section 2, mais formalisé et adapté à notre cadre de calcul sémantique.

On modélise les comparatifs sous la forme de structures prédicat-argument interconnectées, les prédicats étant les principaux opérateurs de comparaison (morphèmes de comparaison implicites et explicites), et les arguments étant connectés aux prédicats via des rôles sémantiques (relations). Notre cadre comprend non seulement des comparaisons explicites, mais également des comparaisons implicites sous forme d'évaluation ou de mesure sur une échelle, qui seront expliquées tout au long de cette section. Vous trouverez une liste plus détaillée et complète des prédicats, des rôles sémantiques et des arguments dans les informations supplémentaires.

\subsection{Prédicats}

20 Le tableau 1 répertorie tous les opérateurs de prédicats et montre qu'il existe quatre principaux types de prédicats: les comparatifs, les extrêmes, les bases et les mesures. La plupart de ces types peuvent être associés à des opérateurs de n'importe laquelle de nos parties du discours : Adjectif (JJ), Adverbe (RB), Nom (NN) et Verbe (VB). L'opérateur de prédicat dans chacun des exemples est en italique. Le type de comparaison inclut 
également les opérateurs $<$ et $=<$, qui sont le contraire des opérateurs $>$ et $>=$ présentés dans le tableau. Il est important de noter que le prédicat 'base positive' est en fait l'opérateur pos implicite (comme indiqué dans la section 2.1 ; toutefois, pour une représentation plus facile, nous le spécifions en indiquant son adjectif ou adverbe correspondant. Il en va de même pour les prédicats de mesure : notre cadre capture également la différence subtile entre la signification de "Marie est [grande] positive" et "Marie a 5 pieds [grande] mesure - explicite". Le plus tôt signifie que Marie est grande selon un certain standard de hauteur dans un contexte, tandis que le dernier signifie que la hauteur de Marie est égale au degré de 5 pieds

\subsection{Rôles sémantiques}

21 Chaque prédicat est caractérisé par ses arguments et chaque argument est connecté aux prédicats par un type de relation (rôle sémantique). Le tableau 2 présente les types de rôles sémantiques possibles pour un prédicat. La figure représente le rôle central d'une structure de comparaison, c'est-à-dire que toute comparaison doit avoir un rôle indiquant l'entité principale évaluée / mesurée / comparée sur une échelle. La forme la plus simple de prédicat comparatif, par exemple, «Paul est plus grand que Pierre", implique deux rôles principaux : Figure (Paul) et Grand (Pierre). Les rôles non essentiels sont principalement associés à des comparaisons non comparatives

\subsection{Arguments}

Chaque rôle pointe vers un argument qui peut avoir différents types, comme indiqué dans le tableau 3. Le type d'argument le plus fréquent est individuel, comme dans 'Paul est plus grand que Pierre'. L'autre rôle notable est Phrase-valeur, qui représente un phénomène de comparaison intéressant.

Dans l'exemple correspondant du tableau, la vitesse de conduite de Paul est explicitement comparée à un point de l'échelle de vitesse à laquelle « il était autorisé ». Ces rôles au sol sont classés en tant que phrase-valeur, une phrase verbale signifiant un point de comparaison à l'échelle, pas une entité individuelle. La figure 1 montre un exemple de structure prédicat-argument dans le cadre sémantique décrit.

Tableau 1 : Les types de prédicats définis dans notre cadre

\begin{tabular}{|l|l|l|}
\hline Type de prédicat & Sous-type & Exemples \\
\hline $\begin{array}{l}\text { Comparatifs: } \\
\begin{array}{l}\text { Comparer à une ou plusieurs } \\
\text { entités. }\end{array}\end{array}$ & $\begin{array}{l}\text { JJ: La voiture était plus moderne que je ne l'avais } \\
\text { imaginé. } \\
\text { RB: Paul a couru plus vite que Sophie. } \\
\text { NN: Plus de biscuits que de gâteaux ont été achetés. } \\
\text { VB: Le café est moins consommé que le thé. }\end{array}$ \\
\cline { 2 - 4 } & $>=$ & $\begin{array}{l}\text { JJ: La pizza est aussi chère que les pâtes. } \\
\text { RB: Les hommes ont couru aussi vite que les } \\
\text { femmes. } \\
\text { NN: Ce collège recrute autant de professeurs }\end{array}$ \\
\hline
\end{tabular}




\begin{tabular}{|c|c|c|}
\hline & & $\begin{array}{l}\text { que nous. } \\
\text { VB: Les athlètes boivent autant que les autres. }\end{array}$ \\
\hline & Superlatif & $\begin{array}{l}\text { JJ: Marie est la plus grande de ses collègues. } \\
\text { RB: Mathieu a parlé le plus fort du groupe. } \\
\text { NN: Les juniors sont les plus dynamiques de tous. } \\
\text { VB: Les pompiers ont couru les plus parmi d'autres. }\end{array}$ \\
\hline \multirow{2}{*}{$\begin{array}{l}\text { Extrême: } \\
\text { Indiquer avoir assez ou trop de } \\
\text { qualité ou de quantité. }\end{array}$} & Excessif & $\begin{array}{l}\text { JJ: Marie est trop grande pour tenir dans le fauteuil. } \\
\text { RB: Pierre a couru trop vite pour se faire prendre. } \\
\text { NN: Il y a trop d'élèves à la fête. } \\
\text { VB: le gamin a trop crié. }\end{array}$ \\
\hline & Assertif & $\begin{array}{l}\text { JJ: Marie est assez intelligente pour accepter l'offre. } \\
\text { RB: la machine fonctionne assez régulièrement. } \\
\text { NN: Il y a suffisamment de professeurs à la fête. } \\
\text { VB: Jacques a passé suffisamment d'interviews pour } \\
\text { faire ses preuves. }\end{array}$ \\
\hline \multirow{2}{*}{$\begin{array}{l}\text { Polarité: } \\
\text { Expression sous forme de base de } \\
\text { qualité +/-. }\end{array}$} & Positif & $\begin{array}{l}\text { JJ: Marie est grande. } \\
\text { RB: Paul parle magnifiquement. }\end{array}$ \\
\hline & Négatif & $\begin{array}{l}\text { JJ: Sophie est courte. } \\
\text { RB: Philippe marche lentement. }\end{array}$ \\
\hline \multirow{2}{*}{$\begin{array}{l}\text { La mesure: } \\
\text { Indiquant une mesure sur une } \\
\text { échelle. }\end{array}$} & Explicite & $\begin{array}{l}\text { JJ: Marie est deux fois plus grande } \\
\text { RB: Philippe roule vite à } 60 \mathrm{mph} \text {. }\end{array}$ \\
\hline & Implicite & $\begin{array}{l}\text { JJ: Marie mesure } 5 \text { pieds. } \\
\text { RB: Philippe roule à } 60 \mathrm{mph} .\end{array}$ \\
\hline
\end{tabular}

\section{Prédiction des structures de comparaison}

Étant donné une phrase d'entrée, nous voulons prédire les opérateurs de prédicats, leurs rôles sémantiques et leurs arguments. Nous décomposons ce problème en trois sous-problèmes :

étiquetage des candidats prédicats à l'aide d'un classificateur à plusieurs classes Pour chaque prédicat, en considérant l'ensemble de toutes les portées d'arguments possibles :

Utiliser un classificateur pour prédire l'étiquette du type de rôle Utiliser un classificateur pour prédire l'étiquette de type d'argument

Dans tout ce qui suit, nous nous référons aux travaux sur l'inférence conjointe avec des contraintes cognitives et culturelles qui pèsent sur la représentation en langue d'un événement : 
"l'évènement" sera ici entendu au sens linguistique, comme l'expression en langue d'un état

ou d'une action, i.e. une prédication (Do et al., 2012 ; Berant et al., 2014).

\subsection{Prédiction des prédicats}

\section{sœurs, des parents et de la racine de la phrase dans la dépendance.}

Les "concepts d'attribut» sont les différentes propriétés qu'un actionneur peut décrire, par exemple, "hauteur» et "épaisseur» sont les attributs de l'adjectif «déformé, dégingandé». Enfin, nous incluons la conjonction de toutes ces fonctionnalités.

\subsection{Prédiction des rôles et des arguments}

Étant donné les prédicats, il convient de nommer le rôle prédicat-argument et de prédire le type d'argument. Nous adoptons ici une approche utilisée pour l'étiquetage des rôles sémantiques (Punyakanok et al., 2008) : à partir d'un prédicat, nous collectons tous les constituants de la phrase pour construire un ensemble d'arguments candidats plausibles. En conséquence, chaque prédicat a un ensemble d'arguments candidats qui doivent être étiquetés avec leurs types d'arguments et être affectés d'un bord de rôle sémantique. Ici, nous formons conjointement deux classificateurs de régression logistique pour prédire le type de rôle sémantique et le type d'argument d'une paire prédicat-argument, en utilisant les fonctions d'identification d'argument de (Punyakanok et al., 2008) et en utilisant l'algorithme de Perceptron à structure moyenne (Collins, 2002).

Tableau 2 : Les types de rôles dans notre cadre

\begin{tabular}{|c|c|c|}
\hline $\begin{array}{l}\text { Type de } \\
\text { relation }\end{array}$ & Description & Exemple \\
\hline Figure & $\begin{array}{l}\text { Le rôle principal étant } \\
\text { comparé à quelque chose autre. }\end{array}$ & - [Lara] est plus grande que l'arbre. \\
\hline Sol & $\begin{array}{l}\text { Le rôle principal contre dont le chiffre est } \\
\text { comparé. }\end{array}$ & - Lara est plus grande que [l'arbre]. \\
\hline $\begin{array}{ll}\text { Degré } & \text { de } \\
\text { différence } & \end{array}$ & $\begin{array}{l}\text { Les rôles "plus" et "fois", } \\
\text { indiquant une différence de degré. }\end{array}$ & $\begin{array}{l}\text { - Pierre est [deux fois] plus grand } \\
\text { que Jim. }\end{array}$ \\
\hline
\end{tabular}




\begin{tabular}{|c|c|c|}
\hline Domaine & $\begin{array}{l}\text { L'expression explicite du domaine / de la } \\
\text { population dans laquelle la comparaison } \\
\text { a lieu }\end{array}$ & $\begin{array}{l}\text { - Marie est la plus intelligente [de } \\
\text { ses camarades de classe]. }\end{array}$ \\
\hline Raison & $\begin{array}{l}\text { La raison associée aux prédicats excessifs et } \\
\text { assertifs }\end{array}$ & $\begin{array}{l}\text { - Paul est trop paresseux [pour se } \\
\text { réveiller]. }\end{array}$ \\
\hline $\begin{array}{l}\text { Degré de } \\
\text { mesure }\end{array}$ & $\begin{array}{l}\text { L'indication principale d'une } \\
\text { mesure }\end{array}$ & - Henry a [5 pieds] de taille. \\
\hline Échelle & L'échelle à laquelle la comparaison a lieu & $\begin{array}{l}\text { - La [hauteur] de la chaise est égale } \\
\text { à la [longueur] du canapé. }\end{array}$ \\
\hline
\end{tabular}

Tableau 3 : Les types d'arguments dans notre cadre

\begin{tabular}{|c|c|c|}
\hline Type d'argument & Sous-type & Exemple \\
\hline $\begin{array}{l}\text { Individuel: } \\
\text { Une entité comparée à d'autres. }\end{array}$ & - & $\begin{array}{l}\text { - [Paul] est plus performant que } \\
\text { Susie. }\end{array}$ \\
\hline $\begin{array}{l}\text { Référence: } \\
\text { Une entité référent dont l'antécédent réel } \\
\text { serait résolu au niveau du discours. }\end{array}$ & - & $\begin{array}{l}\text { - Paul est « } 2 \text { fois » plus grand } \\
\text { que [celui]. }\end{array}$ \\
\hline $\begin{array}{l}\text { Phrase: } \\
\text { Introduit un degré sur l'échelle. }\end{array}$ & Valeur & $\begin{array}{l}\text { - Paul conduisait plus vite que [il } \\
\text { était autorisé]. }\end{array}$ \\
\hline $\begin{array}{l}\text { Montant: } \\
\text { L'expression de la quantité dans une } \\
\text { mesure. }\end{array}$ & $\begin{array}{l}\text { Valeur } \\
\text { Très bas-Bas Haut- } \\
\text { Très Haut }\end{array}$ & $\begin{array}{l}\text { Marie est (5 pieds).grande } \\
\text { Marie est deux fois plus grande } \\
\text { que Bill. } \\
\text { Marie est [un peu] plus grande } \\
\text { que Paul. } \\
\text { Marie est [beaucoup] plus } \\
\text { grande que Bill. }\end{array}$ \\
\hline $\begin{array}{l}\text { Lié: } \\
\text { Une limite / approximation étant définie } \\
\text { sur le montant }\end{array}$ & Exact Approximatif & $\begin{array}{l}\text { Marie a [exactement] } 5 \text { pieds de } \\
\text { hauteur. } \\
\text { Pierre était environ trois fois } \\
\text { plus rapide que les autres. }\end{array}$ \\
\hline exprimé. & $\begin{array}{l}\text { Plus bas } \\
\text { Plus haut }\end{array}$ & $\begin{array}{l}\text { Paul marche [au moins] deux } \\
\text { fois plus vite que toi. } \\
\text { Marie est [au plus] deux fois } \\
\text { plus intelligente } \\
\text { que les autres. }\end{array}$ \\
\hline
\end{tabular}




\begin{tabular}{|c|c|c|}
\hline Échelle: & Explicite & $\begin{array}{l}\text { La [hauteur] du pont est trop } \\
\text { basse pour le fourgon. }\end{array}$ \\
\hline L'échelle sur laquelle la mesure est faite. & Implicite & $\begin{array}{l}\text { Pierre est plus [disponible] que } \\
\text { Paul. }\end{array}$ \\
\hline
\end{tabular}

Des systèmes capables de comprendre et de raisonner par rapport aux concurrents sont essentiels pour diverses applications de la programmation neurolinguistiques PNL, allant de la réponse à une question à domaine ouvert à l'analyse du produit. Comprendre les structures comparatives nécessite un cadre sémantique pouvant représenter leur signification sous- jacente. Nous avons essayé de présenter un autre cadre sémantique permettant de représenter le sens de diverses constructions de comparaison en langage naturel, et ce principalement par la modélisation des comparaisons sous forme de paires prédicat-argument reliées par des rôles sémantiques. Cette programmation prend en charge toutes les parties possibles du discours et une variété de mesures et de comparaisons, fournissant ainsi une représentation informatique unique de la sémantique sous-jacente de la comparaison.

Enfin, nous pensons que cette approche logico-sémantique sur les constructions de comparaison nous permet de faire du raisonnement logique et de déduire des comparatifs, et qu'à l'avenir, nous prévoyons de concevoir une tâche de compréhension de la lecture dans laquelle serait utilisé ce cadre pour répondre aux questions de comparaison à partir d'un paragraphe contenant diverses comparaisons interdépendantes.

\section{BIBLIOGRAPHIE}

ALLEN, J., SWIFT, M., DE BEAUMONT, W., « Analyse sémantique profonde du texte », Actes de la conférence 2008 sur la sémantique dans le traitement de texte, Stroudsburg, 2008.

BACRY, P., Les Figures de style et autres procédés stylistiques, Paris, Armand Colin, coll. » Collection Sujets », 1992.

BAKHSHANDEH, O., ALLEN, J., « Des gloses adjectivales aux concepts d'attributs : apprendre différents aspects qu'un adjectif peut décrire », Actes de la XXIe Conférence internationale sur la sémantique numérique, Londres, (IWCS), 2015.

BERANT, J., LIANG, P.,» Analyse sémantique via la paraphrase », 52e conférence annuelle de l'Association pour la linguistique computationnelle (ACL), Blatimore,2014.

BERANT, J., et al., « Modélisation des processus biologiques pour la compréhension en lecture ", Actes de la conférence de sur les méthodes empiriques en traitement de langage naturel (EMNLP 2014), Doha, 2014. 
BOS, J., « Analyse sémantique à large couverture avec boxer », Dans BOS, J., et DELMONTE, R., rédacteurs en chef, Semantics in Text Processing, Actes de la conférence STEP 2008, Recherche en sémantique informatique, Venise, Collège Publications, 2008.

BRESNAN, J., « Syntaxe de la construction de la clause comparative en anglais », in Linguistic Inquiry, vol. 4, No. 3 , Cambridge, MIT Press, 1973.

COHEN, J., « La comparaison poétique : Essai de systématique », in Langages, no 12, Paris, Didier Larousse, 1968.

Collins, M., « Méthodes de formation discriminantes pour les modèles de Markov cachés : théorie et expériences avec les algorithmes de perceptron », Dans Actes de la conférence ACL-02 sur les méthodes empiriques de traitement du langage naturel - Volume 10, Stroudsburg, EMNLP '02, 2008.

CRESSWELl, M., «La sémantique de degré », In HALl PARTEE, B., (ed.), Montague grammar, 261-292, New York, Academic Press, 1976.

GREVISSE, M., Le bon usage, 13e édition refondue par GoosSE, A., Paris - Louvain-la-Neuve, DeBoeckDuculot, 1993.

HEBOYAN, E., La Figure de la comparaison, Artois Presses Université, coll. » Langues et civilisations étrangères ", 2010.

HEIM, I., « Little », Dans Actes de la 16e Conférence sur la sémantique et la théorie linguistique, Université Cornell, Ithaca, 2007.

JINDAL, N., et LIU, B., «Identifier des phrases comparatives dans les documents texte », Actes de la XXIXe conférence internationale annuelle ACM SIGIR sur la recherche et le développement en matière de recherche documentaire, New York, SIGIR, 2006.

KENNEDY, C., «Flou et grammaire : sémantique d'adjectifs relatifs et absolus », in Linguistique et philosophie, 30 (1), 2007.

KESSLER, W., et KUHN, J., « Détecter les expressions de sentiment comparé - Étude de cas dans les commentaires de conception d'annotation », Dans Actes de KONVENS, Allemagne, Hildesheim, 2014.

MORINET C., « La comparaison en amont ou en aval de la métaphore », in Faits de langues, $n^{\circ}$ 5, 1995.

PETIBON, N., » La figuration de la comparaison, une virtualité fictionnelle », dans Colloque 2007 Figure et figuration, Rusca, 2007.

ROBRIEUX, J.-J., Éléments de rhétorique et d'argumentation, Paris, Dunod, 1993.

SOLT, S., «Échelles de mesure en langage naturel », in Langue et Boussole linguistique, 9 (1), Berlin, 2015.

Stechow, A. V., « Comparaison des théories sémantiques de la comparaison », in Journal of Semantics, 3 (1), Oxford, 1984.

WELLWOOD, A., « Hacquard, Valentine, et Pancheva, Roumyana, Mesurer et comparer des individus et des événements ", in Journal of Semantics, 29 (2), Oxford, 2012.

$\mathrm{XU}, \mathrm{K} .$, et all, « Exploitation d'opinions comparatives à partir d'avis de clients concernant la veille concurrentielle », in Decision Support Systems, 50 (4), Harvard, 2011. 


\section{RÉSUMÉS}

Dans cet article, nous nous intéressons à la syntaxe des comparatives, et plus particulièrement à l'analyse sémantique des structures phrastiques exprimant la comparaison. Toutefois les comparatives semblent être un domaine de recherche très délicat en analyse syntaxique, le cadre minimaliste de la grammaire générative a proposé une analyse des traits morphologiques propres aux catégories et à leurs réalisations lexicales, ce qui permet de contrôler l'acceptabilité des configurations structurelles en syntaxe en se référant à leurs structures sémantiques; l'objectif de cette étude est de fournir une telle analyse et de permettre une meilleure compréhension de la formation des structures comparatives. Par la modélisation des comparaisons sous forme de paires prédicat-argument reliées par des rôles sémantiques, nous allons essayer de présenter un autre cadre sémantique permettant de représenter le sens de diverses constructions de comparaison en langage naturel.

In this paper, we are interested in the syntax of comparatives, and more particularly in the semantic analysis of sentence structures expressing the comparison. Comparatives, however, seem to be a very delicate field of research in syntactic analysis. The minimalist framework of generative grammar has proposed an analysis of the morphological traits specific to categories and their lexical realizations, which makes it possible to control the acceptability of structural configurations by syntax by referring to their semantic structures; the objective of this study is to provide such an analysis and to allow a better understanding of the formation of comparative structures. By modeling comparisons in the form of predicate-argument pairs linked by semantic roles, we will try to present another semantic framework for representing the meaning of various natural language comparison constructs.

\section{INDEX}

Mots-clés : structure comparative, rôle sémantique, prédicat-argument, approche logicolinguistique

Keywords : comparative structure, semantic role, predicate-argument, logico-linguistic approach

\section{AUTEUR}

\section{AMOR TAHAR}

Université Larbi Tébessi -Tébessa, Algérie 